\title{
Composição e atividades antioxidante e antimicrobiana dos óleos essenciais de cravo-da-índia, citronela e palmarosa
}

\author{
SCHERER, R. ${ }^{1 *}$; WAGNER, R. ${ }^{2}$; DUARTE, M.C.T. ${ }^{3}$; GODOY, H.T. ${ }^{4}$ \\ Tommasi Analítica LTDA, Rua Luciano das Neves, 2016, CEP: 29107-010, Vila Velha-Brasil. * rodrigo@ \\ tommasianalitica.com.br; 'Departamento de Tecnologia e Ciência dos Alimentos, Centro de Ciências Rurais, \\ Universidade Federal de Santa Maria, Avenida Roraima, 1000, CEP: 97105-900, Santa Maria-Brasil. \\ rogerw@fea.unicamp.br; ${ }^{3}$ Centro Pluridisciplinar de Pesquisas Químicas, Biológicas e Agrícolas, Caixa Postal \\ 6171, CEP: 13081-970, Campinas-Brasil.mduarte@cpqba.unicamp.br; ${ }^{4}$ Faculdade de Engenharia de Alimentos, \\ Departamento de Ciência de Alimentos, Universidade Estadual de Campinas (UNICAMP), Caixa Postal 6121, \\ CEP: 13083-862, Campinas-Brasil. helena@fea.unicamp.br.
}

\begin{abstract}
RESUMO: Foram avaliadas a ação antioxidante, ação antimicrobiana e a composição dos óleos essenciais de cravo-da-índia (Caryophillus aromaticus L.), citronela (Cymbopogon winterianus) e palmarosa (Cymbopogon martinii). A ação antioxidante foi avaliada pelo método de DPPH (2,2difenil-1-picril-hidrazila), e a ação antimicrobiana determinada pelo método da microdiluição definindo-se a concentração inibitória mínima para os microrganismos Staphylococcus aureus, Escherichia coli, Salmonella Thyphimurium, Pseudomonas aeruginosa e Clostridium perfringens. A composição química dos óleos foi determinada por CG-DIC e a identificação dos compostos voláteis por CG-EM, em ambos os casos, utilizando uma coluna capilar DB-5. O óleo de cravoda-índia apresentou uma forte atividade antioxidante e ação antimicrobiana moderada a forte, sendo o eugenol o componente majoritário do óleo de cravo-da-índia (83,7\%). Por outro lado, as amostras de citronela e palmarosa apresentaram fraca ação antioxidante, porém a ação antimicrobiana foi moderada a forte.
\end{abstract}

Palavras-chave: radicais livres, cravo-da-índia, óleos essenciais, antioxidantes

\begin{abstract}
Composition and antioxidant and antimicrobial activities of clove, citronella and palmarosa essential oils. Antioxidant and antimicrobial activities, as well as the chemical composition of essential oils of clove (Caryophillus aromaticus L.), citronella (Cymbopogon winterianus) and palmarosa (Cymbopogon martinii) were studied. Antioxidant activity was evaluated by DPPH (2,2-diphenyl-1-picrylhydrazyl) method and the antimicrobial activity determined by the microdilution method. The minimal inhibitory concentration was defined against Staphylococcus aureus, Escherichia coli, Salmonella Thyphimurium, Pseudomonas aeruginosa and Clostridium perfringens. The chemical composition of oils was determined by GC-FID and volatile compounds were identified by GC-MS, using a DB- 5 fused silica capillary column. Clove essential oil showed very high antioxidant activity and moderate to high antimicrobial activity, and eugenol was its major compound (83.7\%). On the other hand, citronella and palmarosa samples presented low antioxidant activity but showed moderate to high antimicrobial activity.
\end{abstract}

Key words: free radicals, clove, essential oils, antioxidants

\section{INTRODUÇÃO}

Óleos essenciais são produtos aromáticos de metabolismo secundário de plantas, normalmente produzidos por células secretoras ou grupos de células, sendo encontrados em diversas partes do vegetal, como folhas e talos. São comumente concentrados em uma região do vegetal, como nas folhas, casca ou frutos, e freqüentemente apresentam composição diferente (Conner, 2003). A aplicação de

Recebido para publicação em 01/12/2008

Aceito para publicação em 23/04/2009

Rev. Bras. PI. Med., Botucatu, v.11, n.4, p.442-449, 2009. 
óleos essenciais como ingredientes funcionais em formulações alimentícias, cosméticas ou ainda em formulações sanitizantes, tem despertado grande interesse neste setor industrial devido à grande aceitação dos consumidores por produtos naturais, bem como pelos danos à saúde propiciados pelos aditivos sintéticos.

A literatura atual apresenta inúmeros relatos sobre atividade biológica de extratos vegetais, como ação antifúngica (Alaniìs-Garza et al., 2007; Korukluoglu et al., 2008), ação antibacteriana (Zhang et al., 2007; Al-Turki et al., 2008), atividade anti-tumoral (Jiménez-Medina et al., 2006; Kaileh et al., 2007), ação antiinflamatória e analgésica (Bose et al., 2007; DliazViciedo et al., 2008) atividade antioxidante (Ferreres et al., 2007; Starzynìska-Janiszewska et al., 2008), dentre outras.

O cravo-da-índia (Caryophillus aromaticus L.) é uma planta amplamente utilizada para as mais diversas aplicações, como, por exemplo, anestesia para peixes durante o transporte ou para minimizar o estresse antes do abate (Roubach et al., 2005), ou ainda como anestésico tópico (Alqareer al., 2006), tratamento de candidíase vaginal (Ahmad et al., 2005), além de ser muito utilizado na culinária. A literatura apresenta algumas publicações atribuindo atividades biológicas ao óleo de cravo-da-índia, tais como antimicrobiana (Nunez et al., 2001; Velluti et al., 2004; Viuda-Martos et al., 2007), antioxidante (Bamdad et al., 2006; Jirovetz et al., 2007; Yanishlieva et al., 2006) e anestésica (Altun et al., 2006; Seol et al., 2007).

Suhr \& Nielsen (2003) estudaram o efeito de óleos essenciais e extratos sobre a deterioração de pão de centeio por fungos. Os resultados mostraram que extratos de capim-limão, canela, cravo, mostarda e tomilho apresentaram efeito positivo na preservação do pão. Mytle et al. (2006) relataram que a aplicação de óleo de cravo na proporção de 1 e $2 \%$ reduziu a contagem de Listeria monocytogenes em salsicha alemã.

As plantas citronela (Cymbopogon winterianus) e palmarosa (Cymbopogon martinii) têm sido estudadas em relação a atividade repelente de insetos (Makhaik et al., 2005; Kumar et al., 2007) e ação antifúngica (Duarte et al., 2005), entretanto, não há relatos sobre a atividade antioxidante. Em outro estudo prévio, Mendonça et al. (2005) relataram que o óleo essencial de citronela mostrou elevada atividade contra larvas do mosquito Aedes Aegypti.

Compostos com propriedades biológicas, produzidos por diversas plantas, podem ser utilizados para síntese de novos medicamentos, ou mesmo, serem utilizados como substitutos de princípios ativos sintéticos, como os antibióticos, no intuito de reduzir a resistência microbiana (Ahmad \& Beg, 2001). Sendo assim, o objetivo do presente trabalho foi investigar as atividades antioxidante e antimicrobiana dos óleos essenciais de cravo-da-índia, citronela e palmarosa, bem como caracterizar os constituintes voláteis das amostras.

\section{MATERIAL E MÉTODO}

Os óleos essenciais de cravo-da-índia (Caryophillus aromaticus L.), citronela (Cymbopogon winterianus) e palmarosa (Cymbopogon martinii) foram adquiridos comercialmente da Dierberger Óleos Essenciais S.A. (Brasil) em embalagens de $5 \mathrm{Kg}$ de onde foram retiradas alíquotas para as análises. Os compostos amoxicilina, enrofloxacina, eritromicina, lincomicina, oxitetraciclina, penicilina $\mathrm{G}$, sulfadiazina, sulfanilamida, tetraciclina, ácido gálico, ácido protocatecuico, ácido clorogênico, ácido caféico, ácido ferúlico e quercetina, BHA (butil-hidroxianisol) e o DPPH (2,2-difenil-1-picril-hidrazila) foram adquiridos da Sigma-Aldrich (EUA).

A análise dos constituintes químicos das amostras foi realizada em um cromatógrafo a gás (Varian 3800) acoplado a um detector de ionização em chama (DIC), em triplicata. Os compostos voláteis foram separados em uma coluna capilar DB-5 (30 m x 0,25 mm d.i. x 0,25 $\mu \mathrm{m}$, J\&W Scientific, Folson Califórnia, USA). A programação de temperatura iniciou a $60^{\circ} \mathrm{C}$, aumentando até $240^{\circ} \mathrm{C}$ por uma rampa de temperatura de $3^{\circ} \mathrm{C} \mathrm{min}^{-1}$, permanecendo por 7 min. Utilizou-se o gás hélio para arraste, com uma vazão constante de $1 \mathrm{~mL} \mathrm{~min}{ }^{-1}$. As temperaturas do injetor e do detector foram mantidas em 230 e $250^{\circ} \mathrm{C}$, respectivamente. As amostras foram diluídas em hexano ( $\left.1 \mathrm{mg} \mathrm{mL}^{-1}\right)$ e o volume injetado foi de $1,0 \mu \mathrm{L}$ com o injetor no modo splitless. As percentagens relativas dos compostos foram calculadas a partir das áreas médias obtidas dos cromatogramas. A série homóloga de alcanos (C6-C19) foi analisada nas mesmas condições cromatográficas e, a partir dos tempos de retenção destes, foram calculados os Índices de Kovats (IK) dos compostos voláteis.

A identificação dos constituintes químicos das amostras foi realizada em um cromatógrafo a gás (Shimadzu 17A) acoplado a um espectrômetro de massas (EM) (Shimadzu QP-5000) nas mesmas condições cromatográficas descritas acima, exceto que a temperatura da interface (CG-EM) foi mantida em $240^{\circ} \mathrm{C}$. O detector de massas foi operado no modo de ionização por elétrons $(+70 \mathrm{eV})$, utilizando o modo de varredura, realizada de 35 a $400 \mathrm{~m} / z$ e a voltagem do detector foi de $1,6 \mathrm{kV}$. A identificação dos compostos foi realizada primeiramente pela comparação da similaridade dos espectros de massa obtidos com os da literatura (NIST 98; Adams, 1995), e posteriormente realizou-se a comparação do Índice Kovats com a literatura (IK) (Adams, 1995). Alguns compostos foram considerados positivamente identificados, quando comparados através dos espectros de massas e tempos de retenção dos 
analitos e dos padrões.

A atividade antioxidante foi determinada pelo método DPPH, segundo Scherer \& Godoy (2009). Foram utilizados $3,9 \mathrm{~mL}$ de solução de DPPH $(0,2$ $\mathrm{mM}$ ) em metanol e $0,1 \mathrm{~mL}$ de amostra ou padrões diluídos em metanol em diferentes concentrações. No branco foram utilizados $3,9 \mathrm{~mL}$ de DPPH $(0,2 \mathrm{mM})$ em metanol e $0,1 \mathrm{~mL}$ de metanol. Após a adição dos extratos, os tubos foram agitados vigorosamente e mantidos em repouso por 90 minutos no escuro. Após esse período, a atividade antioxidante foi medida em um espectrofotômetro $(\lambda=517 \mathrm{~nm})$, previamente calibrado com metanol puro. Todas as análises foram realizadas em triplicata. O índice DPPH foi calculado através da equação $1:$ I $(\%)=\left[\left(\mathrm{Abs}_{0}-\mathrm{Abs}_{1}\right) / \mathrm{Abs}_{0}\right] \mathrm{x}$ 100 (1), onde Abs $_{0}$ é a absorbância do branco e Abs, é a absorbância da amostra. $\mathrm{O} I \mathrm{IC}_{50}$ (quantidade suficiente para $50 \%$ de inibição) foi calculado através da equação da reta obtida da curva de calibração (concentração versus o índice DPPH correspondente). A ação antioxidante dos extratos foi expressa pelo Índice de Atividade Antioxidante (IAA), onde tanto a massa de DPPH quanto a massa do extrato utilizada no ensaio foram consideradas para gerar a constante. O IAA é calculado pela equação 2: IAA = massa de DPPH $\left(\mu \mathrm{g} \mathrm{mL}^{-1}\right) / \mathrm{IC}_{50}\left(\mu \mathrm{g} \mathrm{mL}^{-1}\right)$ (2). Considerou-se ação antioxidante fraca quando o IAA $<0,5$, ação moderada quando o AAl estiver entre 0,5 e 1,0 , ação antioxidante forte quando o IAA for de 1,0 a 2,0, e ação muito forte para valor de IAA $>2,0$. Todas as soluções das amostras e padrões, bem como as soluções de DPPH, foram preparadas diariamente antes da utilização.

Para a determinação da atividade antimicrobiana, as amostras e os padrões antibióticos foram diluídos em Dimetilsulfóxido (DMSO) e armazenados $-20^{\circ} \mathrm{C}$ até o momento das análises. $\mathrm{A}$ determinação da concentração inibitória mínima (CIM) foi realizada em placas de cultura com 96 poços, segundo as normas do NCCLS (National Committee of Laboratory Standards, 2003). Os microrganismos utilizados foram Staphylococcus aureus (ATCC 6538), Escherichia coli (isolada de suínos), Salmonella Thyphimurium (ATCC 14028), Pseudomonas aeruginosa (ATCC 13388) e Clostridium perfringens (ATCC 1324). A concentração final de células, ajustada em espectrofotômetro pela escala de McFarland 0,5 , foi na ordem de $10^{4}$ UFC mL. Em cada poço foi adicionado meio de cultura (MuellerHinton 2,1\%), amostra ou o antibiótico, e o inóculo. As concentrações finais avaliadas dos extratos foram de $1,8,1,5,1,2,1,0,0,8,0,6,0,4$ e $0,2 \mathrm{mg} \mathrm{mL}^{-1}$, enquanto que para os antibióticos foram de 250 a $1,95 \mu \mathrm{g} \mathrm{mL}^{-1}$, diluídos em série. Foram incluídos nos testes, controles para verificar a esterilidade do meio, do extrato e da viabilidade do microrganismo. Após a adição do inóculo, as placas foram incubadas a $36^{\circ} \mathrm{C}$ por $24 \mathrm{~h}$, e posteriormente adicionado $50 \mu \mathrm{L}$ de cloreto de trifenil tetrazolium (CTT) $(0,5 \%$ em solução aquosa). Após mais $4 \mathrm{~h}$ de incubação, a CIM foi determinada como a menor concentração capaz de inibir o crescimento visível de células, conferido pelo CTT (células mortas não são coradas).

A análise estatística foi realizada utilizando o software Statistic 6.0. As diferenças foram consideradas significativas quando $\mathrm{p}<0,05$ (ANOVA/ teste de Tukey).

\section{RESULTADO E DISCUSSÃO}

Na composição volátil do óleo essencial de cravo-da-índia, três compostos foram considerados majoritários, destacando-se o eugenol, com 83,75\% da área total, seguido pelo $\beta$-cariofileno, com $10,98 \%$, e com 1,26\%, o $\alpha$-humuleno. Outros compostos também foram detectados em quantidades diminutas (Tabela 1). Os resultados presentes foram similares aos encontrados por outros pesquisadores, como Tomaino et al. (2005) que encontraram 82 e $7 \%$ de eugenol e $\beta$-cariofileno, respectivamente, e Snoussi et al. (2008) que encontraram $88 \%$ de eugenol. No óleo essencial de citronela os compostos majoritários foram o $\beta$-citronelal $(45 \%)$, geraniol $(20,71 \%)$ e $\beta$ citronelol (14,49\%), além de outros compostos com área superior a 1\% (Tabela 1). No óleo essencial de palmarosa, o geraniol constituiu mais de $80 \%$, sendo o acetato de geranila o segundo composto em maior percentagem (12\%) (Tabela 1), concordando com Rao et al. (2005) onde foram encontrados teores de 78 $85 \%$ de geraniol e $3,0-12 \%$ de acetato de geranila em diferentes amostras de óleo essencial de palmarosa.

Em estudo prévio, foi encontrado no óleo essencial de citronela o linalol $(27,4 \%)$, citronelol $(10,9 \%)$, geraniol $(8,5 \%)$, cis-calameneno $(4,3 \%), \beta$ elemeno (3,9\%) dentre outros compostos (Malele et al., 2007). Duarte et al. (2005) avaliaram a composição dos óleos essenciais de citronela e palmarosa, e diferentemente dos resultados determinados neste estudo, os autores encontraram para o óleo de citronela $36 \%$ de $\beta$-citronelal, $18 \%$ de $\beta$-citronelol e $11 \%$ de geraniol, enquanto que no óleo de palmarosa, o geraniol apresentou $63 \%$ da área total e o acetato de geranila $28 \%$.

A composição e a concentração dos componentes dos óleos essenciais, de plantas de mesma espécie, podem variar devido a fatores ecológicos e as condições edafoclimáticas (Yin, 1991). Assim, estas diferenças químicas existentes entre os óleos essenciais podem refletir diretamente nas propriedades funcionais, como a atividade antimicrobiana e antioxidante. Em outro estudo prévio, os componentes do óleo essencial de citronela foram afetados pela época de coleta e espaçamento entre 
TABELA 1. Composição química dos óleos essenciais de cravo, citronela e palmarosa, determinadas por CG-DIC.

\begin{tabular}{cccccc}
\hline Composto & $\mathrm{IK}^{*}$ & $\mathrm{IK}^{* *}$ & $\begin{array}{c}\text { CRA } \\
(\%)\end{array}$ & $\begin{array}{c}\text { CIT } \\
(\%)\end{array}$ & $\begin{array}{c}\text { PAL } \\
(\%)\end{array}$ \\
\hline Campfeno & 954 & 952 & - & - & - \\
$\beta$-Mirceno & 994 & 994 & - & - & 0,08 \\
Limoneno & 1032 & 1031 & - & 3,37 & - \\
Eucaliptol & 1036 & 1035 & - & - & - \\
cis-Ocimeno & 1041 & 1040 & - & - & 0,15 \\
trans-Ocimeno & 1051 & 1050 & - & - & 1,52 \\
NI & 1101 & 1100 & - & 0,54 & 1,55 \\
NI & 1149 & 1148 & - & 2,17 & - \\
$\beta$-Citronelal & 1159 & 1156 & - & 45,00 & - \\
$\beta$-Citronelol & 1232 & 1231 & - & 14,49 & - \\
Chavicol & 1255 & 1256 & 0,33 & - & - \\
Geraniol & 1259 & 1260 & - & 20,71 & 81,22 \\
Geranial & 1273 & 1273 & - & 0,67 & 0,53 \\
Acetato de citronelila & 1357 & 1355 & - & 1,46 & - \\
Eugenol & 1365 & 1363 & 83,75 & - & - \\
$\alpha$-Copaeno & 1378 & 1377 & 0,24 & - & - \\
Acetato de geranila & 1386 & 1385 & - & 1,40 & 12,80 \\
$\beta$-Elemeno & 1393 & 1392 & - & 1,50 & - \\
$\beta$-Cariofileno & 1420 & 1420 & 10,98 & - & 0,86 \\
$\alpha$-Humuleno & 1455 & 1454 & 1,26 & - & - \\
$\gamma$-Muroleno & 1478 & 1477 & 0,21 & 0,19 & - \\
germacreno D & 1482 & 1481 & - & 0,57 & - \\
$\alpha$-Muroleno & 1500 & 1499 & - & 0,38 & - \\
$\gamma$-Cadineno & 1515 & 1513 & - & 0,46 & - \\
$\Delta$-Cadineno & 1525 & 1524 & 0,40 & 1,27 & - \\
Elemol & 1551 & 1550 & - & 1,24 & - \\
Butanoato de geranila & 1563 & 1562 & - & - & 0,15 \\
Germacreno D-4-ol & 1577 & 1575 & & 0,25 & - \\
Total & & & 97,17 & 95,67 & 98,86 \\
\hline
\end{tabular}

IK: Índice de Kovats calculado; ${ }^{*}$ CG-EM, ${ }^{* *}$ CG-DIC; NI: não identificado; CIT: óleo de citronela; PAL: óleo de palmarosa; CRA: óleo de cravo.

as plantas, sendo que os constituintes citronelol e citronelal foram significativamente mais elevados em plantas cultivadas com espaçamento intermediário e colhidas após seis meses do plantio, e com $30 \mathrm{~cm}$ de altura, por outro lado, o teor de geraniol foi superior em plantas colhidas quatro meses após o plantio, porém não houve influência do espaçamento e de altura de corte sobre o seu teor (Marco et al., 2007). Esses resultados concordam com Blank et al. (2007), que reportaram que os componentes do óleo essencial de citronela foram alterados conforme a estação do ano e o horário em que a planta foi coletada.

$\mathrm{Na}$ determinação do $\mathrm{IC}_{50}$ foram elaboradas curvas de calibração para cada composto, pois o $\mathrm{IC}_{50}$ deve ser calculado em intervalo de linearidade do índice DPPH. Os compostos fenólicos empregados neste estudo serviram como parâmetro de equivalência de atividade antioxidante aos óleos essenciais testados. $O$ ácido gálico apresentou o maior valor de IAA seguido pelo ácido protocatecuico e quercetina 
$(p<0,05)$. Não houve diferença significativa no valor de IAA, entre o ácido clorogênico, óleo essencial de cravo-da-índia, ácido caféico e BHA, entretanto, apresentaram IAA significativamente maior do que a rutina e o ácido ferúlico (Tabela 2). O óleo essencial de cravo-da-índia apresentou ação antioxidante muito forte provavelmente devido ao elevado teor de eugenol na composição, pois segundo Scherer \& Godoy (2009) o eugenol apresenta valor de IAA entre $10 \mathrm{e}$ 11. Por outro lado, o óleo de citronela apresentou valor de $\mathrm{IC}_{50}$ muito alto e conseqüentemente valor de IAA muito baixo indicando fraca ação antioxidante $(\mathrm{IAA}<0,5)$ e o óleo de palmarosa não apresentou habilidade de reduzir o DPPH, mesmo na maior concentração final avaliada $\left(800 \mu \mathrm{g} \mathrm{mL}^{-1}\right)$.

Estruturalmente, os compostos fenólicos consistem em anel aromático ligado a um ou mais grupos hidroxila, ocorrendo desde simples fenólicos até moléculas altamente polimerizadas (Bravo, 1998). A estrutura dos compostos fenólicos é fator determinante para a atividade anti-radical livre e quelante de metais, assim conhecido como relação estrutura-atividade (Balasundram et al., 2006). A ação antioxidante dos compostos fenólicos ácidos aumenta com o grau de hidroxilação, como no caso do ácido gálico (trihidroxilado), que apresentou maior IAA do que o ácido protocatecuico (dihidroxilado) (Tabela 2). A substituição de um grupo hidroxila no anel aromático por grupo metoxil reduziu a atividade antioxidante do composto ácido ferúlico em relação ao ácido caféico (Tabela 2), fato também reportado por Rice-Evans et al. (1996). Isso pode ser explicado pela redução da capacidade de doação de átomos de hidrogênio da molécula.

A relação estrutura-atividade em flavonóides é geralmente mais complicada do que nos ácidos hidroxibenzóicos e hidroxicinâmicos, devido à maior complexidade das moléculas. Van Acker et al. (1996) relataram que o grau de hidroxilação, bem como a posição dos grupos hidroxilas influenciam na atividade antioxidante dos flavonóides. Além disso, uma dupla ligação combinada com um radical-OH no anel central aumenta a ação antioxidante. Por esse motivo, a quercetina apresentou valor de IAA significativamente maior em relação à rutina, onde o radical-OH do anel central foi substituído por açúcar (rutinose).

$\mathrm{Na}$ Tabela 3 estão apresentados os valores de CIM das amostras e dos antibióticos. O óleo de cravo-da-índia apresentou melhor atividade antimicrobiana nos microrganismos $S$. aureus, E. coli e $P$. aeruginosa, sendo que os microrganismos $S$. Thyphimurium e $C$. perfringens foram os mais resistentes. Os microrganismos E. colie P. aeruginosa foram mais sensíveis ao óleo de citronela do que os microrganismos $S$. aureus, $S$. Thyphimurium e $C$. perfringens. Por outro lado, o óleo de palmarosa apresentou maior eficácia contra os microrganismos S. aureus, E. colie S. Thyphimurium (Tabela 3).

Os resultados do presente trabalho foram similares aos encontrados por Oussalah et al. (2007), onde o óleo essencial de citronela (Vietnam) apresentou valores de CIM de 0,8, 0,4 e 0,05 $\mathrm{mg} \mathrm{mL}^{-1}$, para E. coli, S. Thyphimurium e $S$. aureus, respectivamente, enquanto que os óleo essenciais de palmarosa (Índia) e cravo-da-índia (Madagascar) apresentaram valores de CIM de 0,2, 0,2e 0,1 $\mathrm{mg} \mathrm{mL}^{-1} \mathrm{e}$ 0,1, 0,1, e 0,05 $\mathrm{mg} \mathrm{mL}^{-1}$, para E. coli, $S$. Thyphimurium e $S$. aureus, respectivamente. A diferença na atividade antimicrobiana, como citado anteriormente, pode ser atribuída às diferenças na composição dos óleos essenciais, pois no óleo de cravo, cujos valores de CIM foram bem menores do que os valores encontrados no presente trabalho, o teor de eugenol e acetato de eugenila foram de 78 e $14 \%$, sendo que

TABELA 2. Índice de atividade antioxidante (IAA) dos óleos essenciais de cravo, citronela, palmarosa e dos compostos fenólicos.

\begin{tabular}{ccc}
\hline & $\mathrm{IC}_{50}$ & $\mathrm{IAA}$ \\
\hline Ácido gálico & $2,83 \pm 0,07$ & $27,1 \pm 0,7^{\mathrm{a}}$ \\
Ácido protocatecuico & $3,8 \pm 0,15$ & $20,2 \pm 0,8^{\mathrm{b}}$ \\
Quercetina & $4,9 \pm 0,56$ & $15,9 \pm 1,7^{\mathrm{c}}$ \\
Ácido clorogênico & $7,4 \pm 0,12$ & $10,3 \pm 0,1^{\mathrm{d}}$ \\
Óleo de cravo & $7,8 \pm 0,65$ & $9,9 \pm 0,8^{\mathrm{d}}$ \\
Ácido caféico & $8,2 \pm 0,31$ & $9,4 \pm 0,3^{\mathrm{d}}$ \\
BHA & $8,2 \pm 0,41$ & $9,3 \pm 0,4^{\mathrm{d}}$ \\
Rutina & $12,1 \pm 0,70$ & $6,4 \pm 0,3^{\mathrm{e}}$ \\
Ácido ferúlico & $14,4 \pm 0,66$ & $5,3 \pm 0,2^{\mathrm{e}}$ \\
Óleo de citronela & $743 \pm 18$ & $0,1 \pm 0,0^{\mathrm{f}}$ \\
Óleo de palmarosa & - & - \\
\hline
\end{tabular}

Os valores apresentados correspondem à média e desvio padrão de triplicatas. Letras diferentes correspondem diferenças significativas $(p<0,05)$. 
TABELA 3: Concentração Inibitória Mínima $\left(\mathrm{CIM}-\mathrm{mg} \mathrm{mL}^{-1}\right)$ dos óleos essenciais de cravo, citronela e palmarosa e dos antibióticos.

\begin{tabular}{cccccc}
\hline & S. aureus & E. coli & P. aeruginosa & S. Thyphimurium & C. perfringens \\
\hline Óleo de citronela & $0,600-0,800$ & $0,400-0,600$ & $0,400-0,600$ & $0,600-0,800$ & $0,600-0,800$ \\
Óleo de palmarosa & $0,400-0,600$ & $0,400-0,600$ & $0,600-0,800$ & $0,400-0,600$ & $0,600-0,800$ \\
Òleo de cravo & $0,400-0,600$ & $0,400-0,600$ & $0,400-0,600$ & $0,600-0,800$ & $0,600-0,800$ \\
Amoxicilina & $*$ & $0,031-0,062$ & $0,062-0,125$ & $0,062-0,125$ & $0,031-0,062$ \\
Enrofloxacina & $*$ & $*$ & $*$ & $0,004-0,008$ & $*$ \\
Eritromicina & $*$ & $*$ & $0,031-0,062$ & $0,031-0,062$ & $0,031-0,062$ \\
Lincomicina & $*$ & $0,031-0,062$ & $0,031-0,062$ & $0,031-0,062$ & $0,031-0,062$ \\
Oxitetraciclina & $*$ & $0,008-0,015$ & $0,062-0,125$ & $0,062-0,125$ & $0,062-0,125$ \\
Penicilina G & $*$ & $0,008-0,015$ & $0,062-0,125$ & $0,062-0,125$ & $0,062-0,125$ \\
Sulfadiazina & $0,062-0,125$ & $0,004-0,008$ & $0,062-0,125$ & $0,062-0,125$ & $0,062-0,125$ \\
Sulfanilamida & $0,062-0,125$ & $0,004-0,008$ & $0,062-0,125$ & $0,062-0,125$ & $0,062-0,125$ \\
Tetraciclina & $*$ & $*$ & $0,031-0,062$ & $0,031-0,062$ & $0,008-0,015$ \\
\hline
\end{tabular}

* $<0,0019 \mathrm{mg} \mathrm{mL}^{-1}$

no presente trabalho o teor de eugenol foi de $85 \% \mathrm{e}$ não foi encontrado acetato de eugenila.

De maneira geral, os microrganismos testados apresentaram menor resistência ao antibiótico enrofloxacina, sendo a $S$. Thyphimurium a bactéria mais resistente a esse antibiótico (Talela 3).

Duarte et al. (2005) estabeleceram, com base no trabalho de Aligiannis et al. (2001), classificação para atividade antimicrobiana de materiais vegetais, de acordo com os resultados de CIM, sendo considerada como forte, moderada e fraca atividade antimicrobiana, óleos ou extratos que apresentem CIM até $0,5 \mathrm{mg} \mathrm{mL}^{-1}$, entre $0,6 \mathrm{e} 1,5 \mathrm{mg} \mathrm{mL}^{-1}$, e acima de $1,6 \mathrm{mg} \mathrm{mL}^{-1}$, respectivamente. Sendo assim, todos os óleos essenciais apresentaram ação antimicrobiana de moderada a forte.

As ações antimicrobiana e antioxidante do óleo de cravo-da-índia podem ser atribuídas ao eugenol, pois o mesmo representa mais de $80 \%$ da composição deste óleo. O geraniol encontrado no óleo de citronela e no óleo de palmarosa tem sido relatado como um composto de elevada atividade antimicrobiana (Pattnaik et al., 1997; Van Zyl et al., 2006; Duarte et al., 2007; Jirovetz et al., 2007), assim como os compostos citronelal e citronelol encontrados no óleo de citronela (Sato et al., 2006; Kordali et al., 2007).

Considerando as propriedades estudadas das amostras de óleos essenciais, pode se propor que, em algumas circunstâncias, como na forma microencapsulada, estas essências podem atuar de forma eficiente como antimicrobianos, assim tornando-se uma alternativa aos antimicrobianos sintéticos. Adicionalmente, o óleo de cravo-da-índia pode ser utilizado também quando há a necessidade de um antimicrobiano e antioxidante, simultaneamente.

\section{CONCLUSÃO}

O óleo de cravo-da-índia apresentou atividade antioxidante muito forte e ação antimicrobiana moderada a forte sobre os microrganismos avaliados. Os óleos essenciais de citronela e palmarosa apresentaram fraca ação antioxidante, porém moderada a forte ação antimicrobiana.

\section{REFERÊNCIA}

ADAMS, R.P. Identification of essential oils components by gas chromatography/quadrupole mass spectroscopy. Illinois (USA): Allured Publishing Corporation, Carol Stream, 1995. 804p.

AHMAD, I.; BEG, A.Z. Antimicrobial and phytochemical studies on 45 Indian medicinal plants again multidrug resistant human pathogens. Journal of Ethnopharmacology, v.74, p.113-23, 2001.

AHMAD, N. et al. Antimicrobial activity of clove oil and its potential in the treatment of vaginal candidiasis. Journal of Drug Targeting, v.13, n.10, p.555-61, 2005.

AL-TURKI, A.I. et al. Chemical and anti-bacterial characterization of aqueous extracts of oregano, marjoram, sage and licorice and their application in milk and labneh. Journal of Food Agriculture and Environment, 
v.6, p.39-44, 2008

ALANliS-GARZA, B.A. et al. Screening of antifungal activity of plants from the northeast of Mexico. Journal of Ethnopharmacology, v.114, p.468-71, 2007.

ALIGIANNIS, N. et al. Composition and antimicrobial activity of the essential oils of two Origanum species. Journal of Agricultural and Food Chemistry, v.40, p.4168-70, 2001

ALQAREER, A. et al. The effect of clove and benzocaine versus placebo as topical anesthetics. Journal of Dentistry, v.34, p.747-50, 2006.

ALTUN, T. et al. Effects of clove oil and eugenol on anesthesia and some hematological parameters of European eel Anguilla anguilla L. Journal of Applied Animal Research, v.30, n.2, p.171-6, 2006

BALASUNDRAM, N. et al. Phenolic compounds in plants and agri-industrial by-products: antioxidant activity, occurrence, and potential uses. Food Chemistry, v.99, p.191-203, 2006.

BAMDAD, F. et al. Evaluation of phenolic content and antioxidant activity of Iranian caraway in comparison with clove and BHT using model systems and vegetable oil. International Journal of Food Science and Technology, v.41, n.1, p.20-7, 2006.

BLANK, A.F. et al. Influence of season, harvest time and drying on Java citronella (Cymbopogon winterianus Jowitt) volatile oil. Brazilian Journal of Pharmacognosy, v.17, p.557-64, 2007.

BOSE, A. et al. Analgesic, anti-inflammatory and antipyretic activities of the ethanolic extract and its fractions of Cleome rutidosperma. Fitoterapia, v.78, p.515-20, 2007. BRAVO, L. Polyphenols: chemistry, dietary sources, metabolism, and nutritional significance. Nutrition Reviews, v.56, p.317-33, 1998.

CONNER, D.E. Naturally occurring compounds. In: DAVIDSON P.;BRANEN A.L. Antimicrobials in foods. New York: Marcel Dekker, Inc. 1993. p.441-68.

DlìAZ-VICIEDO, R. et al. Modulation of anti-inflammatory responses by diterpene acids from Helianthus annuus $\mathrm{L}$. Biochemical and Biophysical Research Communications, v.369, p.761-6, 2008.

DUARTE, M.C.T. et al. Anti-Candida activity of Brazilian medicinal plants. Journal of Ethnopharmacology, v.97, p.305-11, 2005.

DUARTE, M.C.T. et al. Activity of essential oils from Brazilian medicinal plants on Escherichia coli. Journal of Ethnopharmacology, v.111, p.197-201, 2007.

FERRERES, F. et al. New C-deoxyhexosyl flavones and antioxidant properties of Passiflora edulis leaf extract. Journal of Agricultural and Food Chemistry, v.55, p.10187-93, 2007.

JIMÉNEZ-MEDINA, E. et al. New extract of the plant Calendula officinalis produces a dual in vitro effect: cytotoxic anti-tumor activity and lymphocyte activation. BMC Cancer, v.6, p.1-14, 2006.

JIROVETZ, L. et al. Purity, antimicrobial activities and olfactoric evaluations of geraniol/nerol and various of their derivatives. Journal of Essential Oil Research, v.19, n.3, p.288-91, 2007.

KAILEH, M. et al. Screening of indigenous Palestinian medicinal plants for potential anti-inflammatory and cytotoxic activity. Journal of Ethnopharmacology, v.113, p.510-6, 2007.
KORDALI, S. et al. Screening of antifungal activities of 21 oxygenated monoterpenes in-vitro as plant disease control agents. Allelopathy Journal, v.19, n.2, p.373-91, 2007.

KORUKLUOGLU, M. et al. Antifungal properties of olive leaf extracts and their phenolic compounds. Journal of Food Safety, v.28, p.76-87, 2008.

KUMAR, R. et al. Evaluation of Cymbopogon martinii oil extract for control of postharvest insect deterioration in cereals and legumes. Journal of Food Protection, v.70, n.1, p.172-8, 2007

MAKHAIK, M. et al. Evaluation of anti-mosquito properties of essential oils. Journal of Scientific and Industrial Research, v.64, n.2, p.129-33, 2005.

MALELE, R.S. et al. Essential oil of Cymbopogon winterianus Jowitt from Tanzania: composition and antimicrobial activity. Journal of Essential Oil-Bearing Plants, v.10, p.83-7, 2007.

MARCO, C.A. et al. Características do óleo essencial de capim-citronela em função de espaçamento, altura e época de corte. Horticultura Brasileira, v.25, p.429-32, 2007.

MENDONÇA, F.A.C. et al. Activities of some Brazilian plants against larvae of the mosquito Aedes aegypti. Fitoterapia, v.76, p.629-36, 2005.

MYTLE, N. et al. Antimicrobial activity of clove (Syzgium aromaticum) oil in inhibiting Listeria monocytogenes on chicken frankfurters. Food Control, v.17, n.2, p.102-7, 2006.

NCCLS. Methods for dilution antimicrobial susceptibility tests for bacteria that grow aerobically. Approved Standard. 6th.ed. Wayne: NCCLS, 2003. (NCCLS document M7-A6).

NUNEZ, L. et al. Antifungal properties of clove oil (Eugenia caryophylata) in sugar solution. Brazilian Journal of Microbiology, v.32, n.2, p.123-6, 2001.

OUSSALAH, M. et al. Inhibitory effects of selected plant essential oils on the growth of four pathogenic bacteria: E. coliO157:H7, Salmonella Typhimurium, Staphylococcus aureus and Listeria monocytogenes. Food Control, v.18, p.414-20, 2007.

PATTNAIK, S. et al. Antibacterial and antifungal activity of aromatic constituents of essential oils. Microbios, v.89, n.358, p.39-46, 1997.

RAO, B.R.R. et al. Chemical profiles of primary and secondary essential oils of palmarosa (Cymbopogon martinii (Roxb.) Wats var. motia Burk.). Industrial Crops and Products, v.21, p.121-7, 2005.

RICE-EVANS, C.A. et al. Structure-antioxidant activity relationships of flavonoids and phenolic acids. Free Radical Biology and Medicine, v.20, n.7, p.933-56, 1996. ROUBACH, R. et al. Eugenol as an efficacious anaesthetic for tambaqui, Colossoma macropomum (Cuvier). Aquaculture Research, v.36, n.11, p.1056-61, 2005.

SATO, K. et al. Antimicrobial effect of transcinnamaldehyde, (-)-perillaldehyde, (-)-citronellal, citral, eugenol and carvacrol on airborne microbes using an airwasher. Biological and Pharmaceutical Bulletin, v.29, n.11, p.2292-4, 2006.

SCHERER, R.; GODOY, H.T. Antioxidant activity index (AAI) by 2,2-diphenyl-1-picrylhydrazyl method. Food Chemistry, v.112, n.3, p.654-8, 2009 
SEOL, D.W. et al. Clove oil as an anaesthetic for common octopus (Octopus minor, Sasaki). Aquaculture Research, v.38, n.1, p.45-9, 2007.

SNOUSSI, M. et al. In-vitro anti-Vibrio spp. activity and chemical composition of some Tunisian aromatic plants. World Journal of Microbiology and Biotechnology, v.24, n.12, p.3071-6, 2008.

STARZYNiSKA-JANISZEWSKA, A. et al. Antioxidant properties of extracts from fermented and cooked seeds of Polish cultivars of Lathyrus sativus. Food Chemistry, v.109, n.2, p.285-92, 2008.

SUHR, K.I.; NIELSEN, P.V. Antifungal activity of essential oils evaluated by two different application techniques against rye bread spoilage fungi. Journal of Applied Microbiology, v.94, p.665-7, 2003.

TOMAINO, A. et al. Influence of heating on antioxidant activity and the chemical composition of some spice essential oils. Food Chemistry, v.89, n.4, p.549-54, 2005. Van ACKER, S.A.B.E. et al. Structural aspects of antioxidant activity of flavonoids. Free Radical Biology and Medicine, v.20, n.3, p.331-42, 1996.
Van ZYL, R.L. et al. The biological activities of 20 nature identical essential oil constituents. Journal of Essential Oil Research, v.18, p.129-33, 2006.

VELLUTI, A. et al. Effect of essential oils of cinnamon, clove, lemon grass, oregano and palmarosa on growth of and fumonisin B-1 production by Fusarium verticilloides in maize. Journal of Science of Food and Agriculture, v.84, n.10, p.1141-6, 2004.

VIUDA-MARTOS, M. et al. Antifungal activities of thyme, clove and oregano essential oils. Journal of Food Safety, v.27, n.1, p.91-101, 2007.

YANISHLIEVA, N.V. et al. Natural antioxidants from herbs and species. European Journal of Lipid Science and Technology, v.108, n.9, p.776-93, 2006.

YIN, H.W. Yield and composition variation of essential oil from leaves of different Cinnamonmum osmophloeum Kanehira clones in Taiwan. Quarterly Journal of Chinese Forestry, n.24, p.83-104, 1991.

ZHANG, Y. et al. Antibacterial lignans and triterpenoids from Rostellularia procumbens. Planta Medica, v.73, n.15, p.1596-9, 2007. 